\title{
Forest-Fire Recognition by Sparse and Collaborative Subspace Clustering
}

\author{
Zhen Ye ${ }^{1 *}$, Yifu Jiang', Shihao Shi', Jiefei Yan', Lin Bai ${ }^{1}$ \\ ${ }^{1}$ School of Electronics and Control Engineering, Chang'an University, Xi'an, China \\ ${ }^{2}$ School of Electronics and Information Engineering, Beijing Jiaotong University, Beijing, China \\ Email: *yezhen525@126.com
}

How to cite this paper: Ye, Z., Jiang, Y.F. Shi, S.H., Yan, J.F. and Bai, L. (2019) Forest-Fire Recognition by Sparse and Collaborative Subspace Clustering. Journal of Applied Mathematics and Physics, 7, 2883-2890.

https://doi.org/10.4236/jamp.2019.711197

Received: October 9, 2019

Accepted: November 24, 2019

Published: November 27, 2019

\begin{abstract}
Traditional forest-fire recognition based on the characteristics of smoke, temperature and light fails to accurately detect and respond to early fires. By analyzing the characteristics of flame, the methods based on aerial image recognition have been widely used, such as RGB-based and HIS-based methods. However, these methods are susceptible to background factors causing interference and false detection. To alleviate these problems, we investigate two subspace clustering methods based on sparse and collaborative representation, respectively, to detect and locate forest fires. Firstly, subspace clustering segments flame from the whole image. Afterwards, sparse or collaborative representation is employed to represent most of the flame information in a dictionary with $I_{1}$-regularization or $l_{2}$-regularization term, which results in fewer reconstruction errors. Experimental results show that the proposed SSC and CSC substantially outperform the state-of-the art methods.
\end{abstract}

\section{Keywords}

Forest Fires, Flame Recognition, Sparse Subspace Clustering (SSC), Collaborative Subspace Clustering (CSC)

\section{Introduction}

Forest fires destroy forests and undergrowth plants, endanger human beings and wildlife, pollute soil and air, affect water cycle, and so on. In order to protect the ecological environment and human security, early fire detection is a necessary step for forest fire warning. Conventional detection methods use the characteristics of smoke, temperature and light to monitor fires. However, these methods can't respond quickly to early fire when the forest environment is bad, and the fires may not be detected immediately if it is far away from the sensors [1]. The 
methods based upon aerial image recognition avoid the adverse effects (e.g. space, height and airflow), by analyzing the characteristics of flame (e.g. color, area and location), to detect and locate forest fires accurately. Arguably, flame recognition has been the key to the success of early fire detection [2].

RGB (red, green, and blue) and HIS (hue, intensity and saturation)-based methods mainly make use of color information in flame recognition recent years [3] [4]. RGB-based methods employ color features to recognize fire areas by segmenting objects in a specified color range in RGB images [5]. In these methods, RGB vector $\boldsymbol{m}$ is used to represent the average color estimation. If the color of some pixels is within a certain range of the vector $\boldsymbol{m}$, these pixels will be recognized and classified. The following comparation method RGB employs the Euclidean distance [6] as the classification range. On the other hand, HIS-based methods employ hue, light intensity and saturation as features for flame recognition. In general, the flame colors are usually yellow, red and orange, and the flame hue is distributed in $0-60$ degrees. Due to the light intensity of background, the flame saturation may be affected, causing interference and false detection [7].

Since the sparse coefficients can result in fewer reconstruction errors and can represent most of the important information in a dictionary, we investigate sparse subspace clustering (SSC) for flame recognition to improve the recognition accuracy. Given a set of pixels drawn from a union of linear or affine subspaces, the task is to find segmentation of forest-fire images. Because each pixel in a union of subspaces can always be written as a linear or affine combination of all other pixels, the representation of pixels in the same subspace can be obtained by searching for the sparsest combination. Thus, a similarity matrix will be established for flame segmentation by sparse subspace clustering. Since the combinatorial $l_{0}$-norm minimization is NP-hard, SSC estimates similarity matrix by imposing $l_{1}$-norm optimization [8]. We also studied collaborative representation clustering (CSC) [9] whose similarity matrix is employed by imposing $l_{2}$ -norm optimization instead of $l_{1}$-norm optimization, which means that the "collaborative" nature is instead of "competitive" nature of forest-fire images.

The main contributions of this paper are summarized as follows. 1) The proposed approaches are unsupervised methods requiring no initialization. 2) Our work is the first one to employ the spare or collaborative representation of vectors in subspace clustering for forest-fire recognition. 3) The proposed approaches, whose objective function is optimized by $l_{1}$-norm optimization or $l_{2}$ -norm optimization fully exploiting the internal relationship among pixels, produces stronger ability of discrimination compared with traditional methods (i.e., RGB and HIS) by conducting experiments on three forest-fire images.

The remainder of this paper is organized as follows. In Section 2, we present the proposed approaches. In Section 3, we provide a description of the three forest-fire data for performance evaluation, as well as a detailed comparison with the state-of-the-art methods. Section 4 concludes the presented work. 


\section{Approach}

The basic idea of subspace clustering is to extract a set of points from linear or affine subspace and to find the segments of data. Since each data point in the union set of a subspace can always be written as a linear or affine combination of other points, the sparse or collaborative expression-based subspace clustering method uses the low-dimensional representation of the data in the high-dimensional space by searching the most sparse or collaborative combination. This enables us to establish a similarity matrix and segment flame by spectral clustering. Since the combinatorial $l_{0}$-norm minimization is NP-hard, the $l_{1}$-norm minimization and $l_{2}$-norm minimization employed in sparse subspace clustering and collaborative subspace clustering, respectively. Thus, spectral clustering method is applied to the similarity graph formed by sparse coefficient or collaborative coefficient to realize the detection and recognition of forest-fire images. Figure 1 shows an example of data drawn from two subspaces, the corresponding adjacency matrix and similarity graph. For each data point, the $l_{1}$-minimization or $l_{2}$-minimization gives subspace-preserving representation, hence the adjacency matrix has a block-diagonal structure and the similarity graph has two connected components. Using spectral clustering methods, we can recover the components of the similarity graph, obtaining the segmentation of forest-fire data.

The proposed sparse subspace clustering (SSC) algorithm will be discussed in the following. Let $\mathbf{X}=\left\{\mathbf{X}_{1}, \ldots, \mathbf{X}_{N}\right\}$ be $N$ subspaces of dimensions $\left\{d_{1}, \cdots, d_{N}\right\}$ embedded in $D$ dimensional space. Consider a given collection of $M$ points $\boldsymbol{\psi}=\left\{\boldsymbol{\psi}_{1}, \ldots, \boldsymbol{\psi}_{M}\right\}$ drawn from the $N$ subspaces. Wherein, $\mathbf{Y}$ can be represented by sparse representation if $\mathbf{Y}$ is a linear combination of at most $K$ ( $K<<N$ ) basis vectors in $\psi$. In practice, the signal is $K$-sparse when it has at most $K$ large nonzero coefficients and the remaining coefficients are very small [10]. We can write $\mathbf{Y}$ as

$$
\mathbf{Y}=\sum_{j=1}^{M} \mathbf{X}_{j} \boldsymbol{\Psi}_{j}
$$

$\mathbf{Y}$ and $\mathbf{X}$ belong to the space domain and the subspace domain, respectively. The idea is based on the observation that each data point can be described as a linear combination of all the other data points in subspace domain.

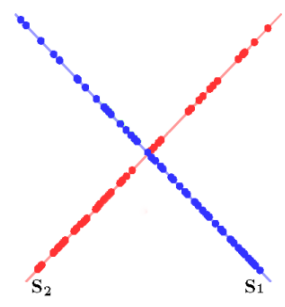

(a)

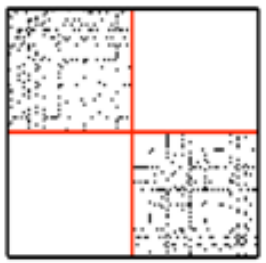

(b)

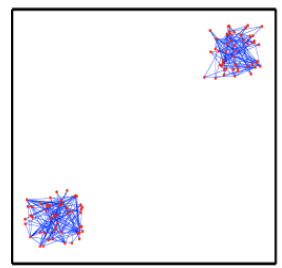

(c)

Figure 1. An example: (a) Data drawn from two subspaces; (b) Corresponding matrix of coefficients; (c) Similarity graph. 
For separating $\mathbf{X}$ into different class-specific representations, we need to calculate

$$
\mathbf{x}_{i}=\mathbf{X} \mathbf{w}_{i}
$$

W is the similarity matrix whose $i^{\text {th }}$ column corresponds to the sparse representation of $\mathbf{x}_{i}$. For each pixel $\mathbf{x}_{i}$ in the dictionary $\mathbf{X}$, the sparse representation vector is calculated by solving the $l_{1}$-norm optimization problem

$$
\min _{\mathbf{w}_{i}}\left\|\mathbf{w}_{i}\right\|_{1} \quad \text { s.t. }\left\|\mathbf{X}_{i} \mathbf{w}_{i}-\mathbf{x}_{i}\right\|^{2} \leq \varepsilon
$$

where $\mathbf{X}_{i}$ does not include $\mathbf{x}_{i}$ itself, $\boldsymbol{w}_{i}=\left[\boldsymbol{w}_{i 1}, \boldsymbol{w}_{i 2}, \cdots, \boldsymbol{w}_{i N}\right]$ is the sparse representation vector corresponding to $\mathbf{x}_{i}$ of size $N \times 1$, and $\varepsilon>0$ is a small tolerance. Thus, the similarity (or affinity) matrix $\mathbf{W}$ is described as

$$
\left(\mathbf{W}_{s}\right)_{i, i^{\prime}}=\left\{\begin{array}{rr}
0, & i=i^{\prime} \\
\mathbf{w}_{i, i^{\prime}}, & i>i^{\prime} \\
\mathbf{w}_{i, i^{\prime}-1}, & i<i^{\prime}
\end{array}\right.
$$

The generalized eigenvalue problem can be expressed as

$$
\min _{\mathbf{w}_{i}}\left\{\left\|\mathbf{x}_{i}-\mathbf{X}_{i} \mathbf{w}_{i}\right\|_{2}^{2}+\lambda\left\|\mathbf{w}_{i}\right\|_{1}\right\}
$$

where $\lambda$ is a Lagrange multiplier balancing the minimization between regularization term and residual part [11]. The procedure of SSC is outlined in Algorithm 1 .

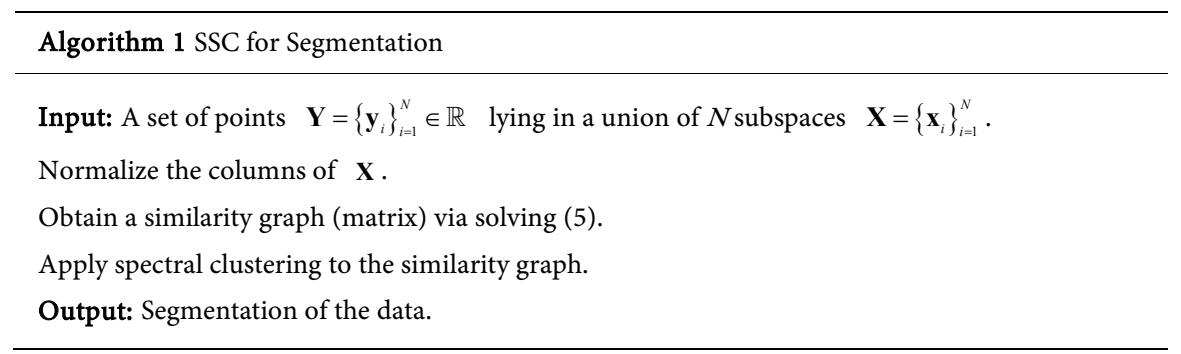

In the next, collaborative subspace clustering (CSC) is also proposed to investigate the collaborative nature of forest-fire images. Collaborative representation is studied to estimate the similarity matrix by replacing the $l_{1}$-norm minimization in solving the weight matrix with an $l_{2}$-norm minimization. The segmentation of forest-fire images is found by applying spectral clustering to a similarity graph formed by the collaborative coefficients. The collaborative representation vector for $\mathbf{x}_{i}$ is calculated by solving the $l_{2}$-norm optimization problem

$$
\min _{\mathbf{w}_{i}}\left\|\mathbf{w}_{i}\right\|_{2} \quad \text { s.t. } \quad \mathbf{X}_{i} \mathbf{w}_{i}=\mathbf{x}_{i}
$$

where $\mathbf{X}_{i}$ does not include $\mathbf{x}_{i}$ itself, and $\mathbf{w}_{i}=\left[\mathbf{w}_{i 1}, \mathbf{w}_{i 2}, \cdots, \mathbf{w}_{i N}\right]$ is the collaborative representation vector corresponding to $\mathbf{x}_{i}$ of size $(N-1) \times 1$. The objective function can be reformulated as

$$
\min _{\mathbf{w}_{i}}\left\{\left\|\mathbf{x}_{i}-\mathbf{X}_{i} \mathbf{w}_{i}\right\|_{2}^{2}+\lambda\left\|\mathbf{w}_{i}\right\|_{2}^{2}\right\}
$$


The regularization parameter $\lambda$ does two things: it makes the least square solution stable; and it introduces a weaker "sparsity" than that by $l_{1}$-norm minimization to the solution depicted as Equation (6). The collaborative representation vector can be calculated as

$$
\mathbf{w}_{i}=\left(\mathbf{X}_{i}^{T} \mathbf{X}_{i}+\lambda \mathbf{I}\right)^{-1} \mathbf{X}_{i}^{T} \mathbf{x}_{i} .
$$

Similarity matrix $\mathbf{W}=\left[\mathbf{w}_{1}, \mathbf{w}_{2}, \cdots, \mathbf{w}_{N}\right]$ represents the graph weight matrix of size $N \times N$ and $\operatorname{diag}(\mathbf{W})$ is a zero vector. The graph weight matrix can be calculated by within-class samples to generate class-specific collaborative coefficients. The procedure of the proposed CSC is outlined in Algorithm 2.

Algorithm 2 CSC for Segmentation

Input: A set of points $\mathbf{Y}=\left\{\mathbf{y}_{i}\right\}_{i=1}^{N} \in \mathbb{R}$ lying in a union of $N$ subspaces $\quad \mathbf{X}=\left\{\mathbf{x}_{i}\right\}_{i=1}^{N}$.

Solve the sparse optimization problem by Equation (7).

Normalize the columns of $\mathbf{X}$.

Obtain a similarity graph (matrix) with $N$ nodes representing the data points by Equation (8).

Apply spectral clustering to the similarity graph.

Output: Segmentation of the data.

\section{Experimental Results}

Experiments are conducted using three forest-fire images, which are represent a forest-fire scenario with $55 \times 59$ pixels, $79 \times 63$ pixels, and $58 \times 51$ pixels. To quantify the efficacy of the proposed two approaches, we study the classification performance of them and two state-of-the-art methods (HIS and RGB) for classifying forest-fire data. Table 1 shows the classification results with the overall classification accuracy (OA). From this table, we can see that the proposed methods are superior to HIS and RGB. In particular, the proposed SSC has more significant advantage in classification accuracy. For example, the OA of SSC has improvements of $10.31 \%$ and $1.05 \%$ for image 1 compared with HIS and RGB, respectively; the OA of SSC has improvements of $3.8 \%$ and $3.32 \%$ for image 2 compared with HIS and RGB, respectively.

Figures 2-4 provide the classification maps of the aforementioned methods for the forest-fire images. From the classification maps, it can be seen that the proposed SSC and CSC have better classification performance of details, which can play a positive role in the assessment of fire severity and areas.

Table 1. Classification results for the four methods.

\begin{tabular}{ccccc}
\hline \multirow{2}{*}{ Image } & \multicolumn{4}{c}{ Overall Classification Accuracy (OA) } \\
\cline { 2 - 5 } & HIS & RGB & SSC & CSC \\
\hline 1 & $77.69 \%$ & $86.95 \%$ & $88.00 \%$ & $87.46 \%$ \\
2 & $86.56 \%$ & $87.04 \%$ & $90.36 \%$ & $87.26 \%$ \\
3 & $84.16 \%$ & $88.22 \%$ & $88.75 \%$ & $88.63 \%$ \\
\hline
\end{tabular}




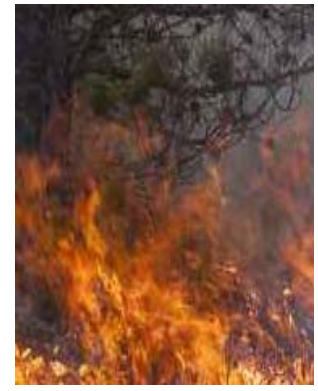

(a)

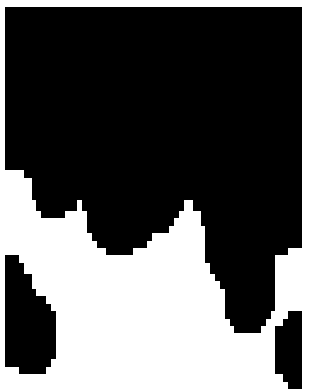

(c)

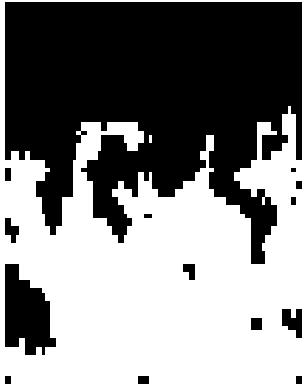

(d)

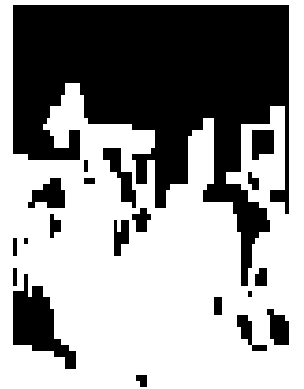

(b)

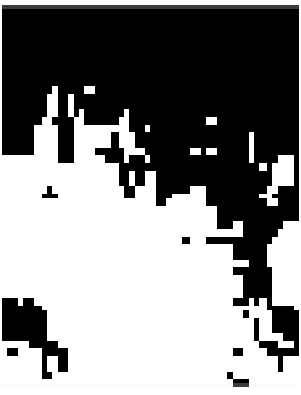

(e)

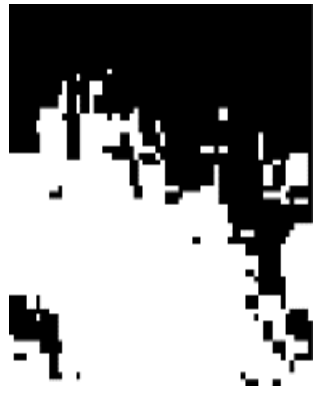

(f)

Figure 2. Classification maps of four approaches for image 1. (a) Image 1; (b) Ground-truth; (c) HIS; (d) RGB; (e) SSC; (f) CSC.

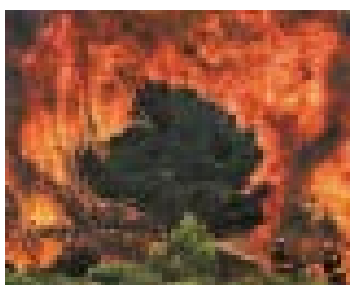

(a)

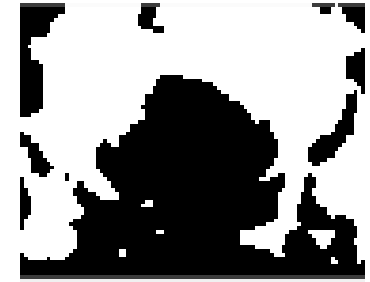

(b)

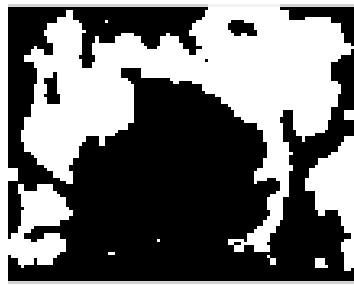

(c)

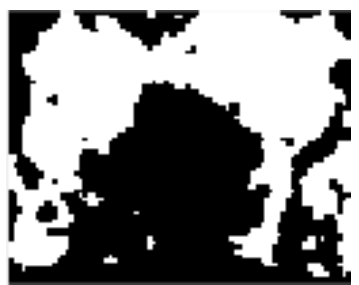

(d)

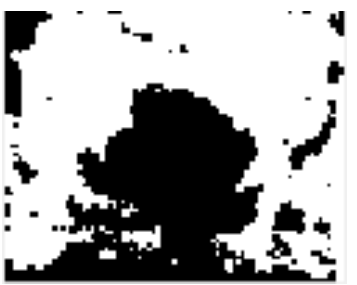

(e)

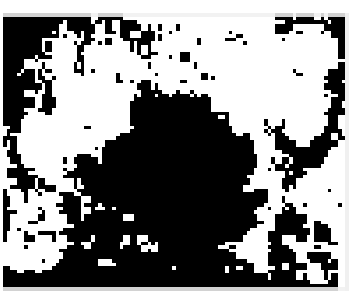

(f)

Figure 3. Classification maps of four approaches for image 3. (a) Image 3; (b) Ground-truth; (c) HIS; (d) RGB; (e) SSC; (f) CSC.

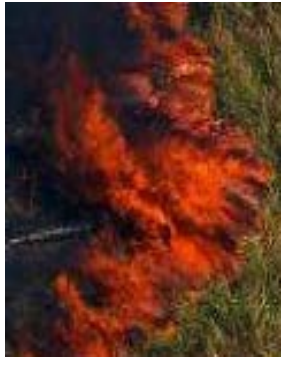

(a)

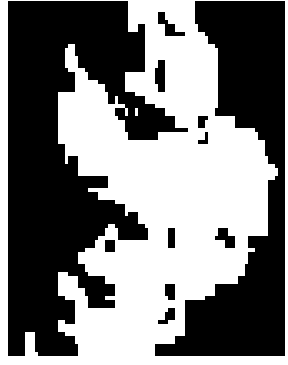

(b) 


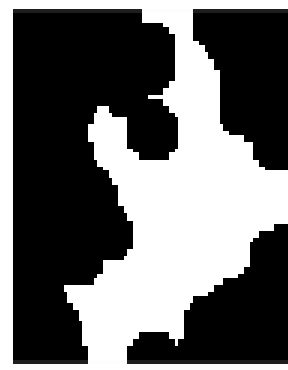

(c)

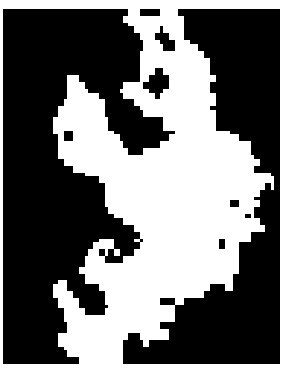

(d)

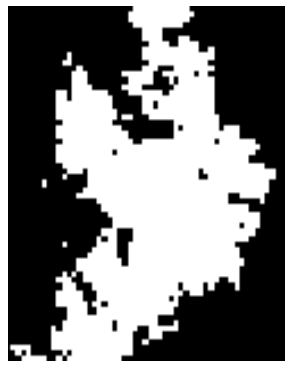

(e)

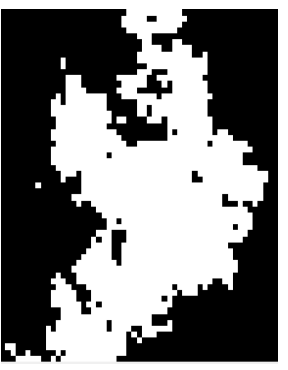

(f)

Figure 4. Classification maps of four approaches for image 2. (a) Image 2; (b) Ground-truth; (c) HIS; (d) RGB; (e) SSC; (f) CSC.

\section{Conclusion}

In this paper, we propose two unsupervised classification methods based on sparse or collaborative representation of vectors in subspace clustering for forest-fire recognition. These strategies employing $l_{1}$-norm or $l_{2}$-norm optimization exploits the internal relationship among pixels and produces stronger ability of discrimination. Experimental results show that the proposed methods substantially outperform the state-of-the-art methods. We hence conclude that the proposed SSC and CSC approach are two effective approaches for forest-fire recognition.

\section{Acknowledgements}

This research was supported by the National Natural Science Foundation of China $(41601344,61803042)$.

\section{Conflicts of Interest}

The authors declare no conflicts of interest regarding the publication of this paper.

\section{References}

[1] Wang, Y.B. and Ma, X.M. (2014) Early Fire Detection for High Space Based on Video-Image Processing. 2014 International Symposium on Computer, Consumer and Control, Taichung, 10-12 June 2014.

[2] Qiu, T., Yan, Y. and Lu, G. (2012) An Autoadaptive Edge-Detection Algorithm for Flame and Fire Image Processing. IEEE Transactions on Instrumentation and Measurement, 61, 1486-1493. https://doi.org/10.1109/TIM.2011.2175833

[3] Wang, L. and Li, A.G. (2017) Early fire recognition based on Multi-Feature Fusion of Video Smoke. The 36th Chinese Control Conference, Dalian, 26-28 July 2017. https://doi.org/10.23919/ChiCC.2017.8028197

[4] Chen, T.-H., Wu, P.-H. and Chiou, Y.-C. (2004) An Early Fire-Detection Method Based on Image Processing. 2004 International Conference on Image Processing, Singapore, 24-27 October 2004.

[5] Gonzalez, R.C. and Woods, R.E. (2017) Digital Image Processing. Publishing House of Electronics Industry, Beijing.

[6] Chamorro-Martines, J., Soto-Hidalgo, J.M., Martinez-Jimenez, P.M., et al. (2017) 
Fuzzy Color Spaces: A Conceptual Approach to Color Vision. IEEE Transaction on Fuzzy Systems, 25, 1264-1280. https://doi.org/10.1109/TFUZZ.2016.2612259

[7] Celik, T. and Demirel, H. (2009) Fire Safety in Video Sequence Using a Generic Color Model. Fire Safety, 44, 951-61. https://doi.org/10.1016/j.firesaf.2008.05.005

[8] Elhamifar, E. and Vidal, R. (2013) Sparse Subspace Clustering: Algorithm, Theory, and Applications. IEEE Transactions on Pattern Analysis and Machine Intelligence, 35, 2765-2781. https://doi.org/10.1109/TPAMI.2013.57

[9] Zhang, Y.B., Zhang, Y.L., Zhang, J. and Dai, Q.H. (2016) CCR: Clustering and Collaborative Representation for Fast Single Image Super-Resolution. IEEE Transactions on Multimedia, 18, 405-417. https://doi.org/10.1109/TMM.2015.2512046

[10] Elhamifar, E. and Vidal, R. (2009) Sparse Subspace Clustering. IEEE Computer Society Conference on Computer Vision and Pattern Recognition, Miami, 20-25 June 2009. https://doi.org/10.1109/CVPR.2009.5206547

[11] Zhang, L., Yang, M. and Feng, X.C. (2011) Sparse Representation or Collaborative Representation: Which Helps Face Recognition? IEEE International Conference on Computer Vision, Barcelona, Spain, November 2011, 471-478. 